\title{
Autophagy in Neurodegenerative Diseases: From Mechanism to Therapeutic Approach
}

\author{
Jihoon $\mathrm{Nah}^{1}$, Junying Yuan ${ }^{2, *}$, and Yong-Keun Jung ${ }^{1, *}$
}

\begin{abstract}
Autophagy is a lysosome-dependent intracellular degradation process that allows recycling of cytoplasmic constituents into bioenergetic and biosynthetic materials for maintenance of homeostasis. Since the function of autophagy is particularly important in various stress conditions, perturbation of autophagy can lead to cellular dysfunction and diseases. Accumulation of abnormal protein aggregates, a common cause of neurodegenerative diseases, can be reduced through autophagic degradation. Recent studies have revealed defects in autophagy in most cases of neurodegenerative disorders. Moreover, deregulated excessive autophagy can also cause neurodegeneration. Thus, healthy activation of autophagy is essential for therapeutic approaches in neurodegenerative diseases and many autophagy-regulating compounds are under development for therapeutic purposes. This review describes the overall role of autophagy in neurodegeneration, focusing on various therapeutic strategies for modulating specific stages of autophagy and on the current status of drug development.
\end{abstract}

\section{INTRODUCTION}

Eukaryotic cells use two major strategies for protein degradation, the ubiquitin-proteasome system and autophagy-lysosome pathway. The latter is a highly conserved, lysosome-dependent degradation process. Autophagy isolates cytosolic materials within a double membrane vesicle called an "autophagosome" which then fuses with lysosome to degrade isolated substrates (Mizushima et al., 2011). Until now, more than 30 autophagy-related (ATG) genes and three types of autophagy, macroautophagy, microautophagy, and chaperone-mediated autophagy (CMA), have been identified in yeast and mammalian systems (Boya et al., 2013; Nakatogawa et al., 2009). Moreover, various forms of selective autophagy

${ }^{1}$ Global Research Laboratory, School of Biological Science, Seoul National University, 1 Gwanak-ro, Gwanak-gu, Seoul 151-747, Korea, ${ }^{2}$ Department of Cell Biology, Harvard Medical School, 240 Longwood Avenue, Boston, MA 02115-5730, USA

*Correspondence: ykjung@snu.ac.kr (YKJ); junying_yuan@hms.harvard.edu (JYY)

Received 6 February, 2015; accepted 9, February, 2015; Published online 20 April, 2015

Keywords: ALS, autophagy, HD, PD, therapeutics have been characterized. Each is involved in the turnover of different cellular organelles (Komatsu and Ichimura, 2010). Defective regulation of the autophagy machinery and/or dysfunction of the lysosomal process can disrupt cellular homeostasis and lead to various disorders (Shintani and Klionsky, 2004). Three major autophagy deregulations have been observed in neurodegenerative diseases: (1) insufficient autophagy activation, (2) autophagy dysfunction due to reduced lysosomal function, and (3) autophagic stress related to pathologic activation of autophagy (Cherra and Chu, 2008). These observations indicate that autophagy generally plays an important neuroprotective role.

In this review, the overall mechanism of autophagy, the role of autophagy in neurodegeneration, and recent strategy for identifying therapeutic drugs will be described. Finally, the current strategies for targeting autophagy and the major issues in therapeutic autophagy for neurodegeneration treatment will be discussed.

\section{SEQUESTRATION OF CYTOSOLIC MATERIALS AND AUTOPHAGOSOME MATURATION}

For many years, autophagy was considered as a non-selective catabolic process to supply amino acids in response to starvation. Nowadays, however, autophagy is considered to recognize selective target proteins under various stress conditions, while a non-selective catabolic process is accepted in some cases (Filimonenko et al., 2010). Macroautophagy is the most widely studied and characterized autophagy process. The cytosolic substrates are sequestered within autophagosomes for lysosomal degradation. In microautophagy, the lysosomal or vacuolar membrane forms invaginations which then are differentiated into the autophagic tube to sequestrate portions of the cytosol for its degradation (Li et al., 2012). During CMA, heat shock cognate (HSC) 70, a chaperone protein, recognizes a pentapeptide, KFERQ, in its cytosolic substrate protein and then binds to a receptor on the lysosomal membrane, LAMP2A. HSC70 finally unfolds these proteins and translocates directly to the lysosome (Kaushik and Cuervo, 2012).

Macroautophagy is initiated through the phosphorylation of the ULK1 complex (ULK1, ULK2, ATG13, FIP200, and ATG101). Depending on cellular energy level, phosphorylation of ULK1 (Ser 758) components, which are involved in the ULK1 complex inhibition, is regulated by mammalian target of rapamycin complex 1 (TORC1), while phosphorylation of ULK1 (Ser 317 and 377) components, involved in ULK1 complex activation, is regulated by AMP-activated protein kinase (AMPK) (Mizushima, 2010). Activated ULK1 complex recruits 
downstream ATG proteins to the autophagosome formation site and phosphorylates beclin 1 (BECN1) on Ser 14, promoting the activity of the VPS34 complex (PI3KCIII, BECN1, ATG14L, and VPS15) to initiate autophagosome formation (Russell et al., 2013). During the nucleation process, the VPS34 complex generates phosphatidylinositol 3-phosphate (PI3P). The accumulation of PI3P provides a platform to recruit PI3P-binding proteins (Shibutani and Yoshimori, 2014). BECN1, a protein essential for the VPS34 complex, has many binding partners such as ATG14L, UVRAG, and BCL2. Through these interacting partners, BECN1 finely regulates autophagy under different stress conditions (Abrahamsen et al., 2012). When the nucleation step starts, the autophagosome membrane is expanded.

In the elongation process, the autophagosome membrane around the cytoplasmic substrates requires two ubiquitin-like systems to extend the membrane. In the Atg5-Atg12 conjugation system, Atg12 was identified as the first ubiquitin-like protein (Ubl). Atg12 is activated by the E1-like enzyme, Atg7, and is transferred to the E2-like enzyme, Atg10, on its target protein Atg5. Finally, Atg5-Atg12 interacts with Atg16 to form a dimeric complex. The Atg5-Atg12-Atg16 complex targets the membranes depending on the formation of PI3P (Shpilka et al., 2012). The second ubiquitin-like conjugation system is the Atg8 conjugation system. Atg8 is cleaved by the cysteine protease, Atg4, and is processed by the ubiquitin-like enzymes, Atg7 and Atg3. The conjugated Atg8-phosphatidylethanolamine (PE) resides on the phagophore membrane and participates in cargo recruitment to the autophagosome (Weidberg et al., 2011). In the maturation process, the cargo sequestration is completed and the autophagosome fuses with lysosomes to degrade its cargo. Recent studies have implicated SNARE proteins, endosomal COPs, ESCRT III complex, small GTPase Rab proteins, chaperone HSP70 family proteins, and TECPR1 as crucial components in autophagosome maturation (Chen et al., 2012; Hyttinen et al., 2013; Nair et al., 2011). In the final step of autophagy, the cargo is broken down and degraded to cellular components and then released into the cytosol.

\section{CHARACTERISTICS OF NEURONAL AUTOPHAGY}

In general, the autophagic process has been reported to protect the neurons. Neuronal autophagy is essential for synaptic plasticity, anti-inflammatory function in glial cells, oligodendrocyte development, and myelination process (Kesidou et al., 2013; Lee, 2012). The aggregate-prone proteins in neurons cannot be diluted by cell division because neurons are post-mitotic cells. Thus, neurons require well-regulated protein quality control systems. Accordingly, altered activity of the protein degradation system causes the accumulation of abnormal proteins and eventually leads to neuronal dysfunction such as deregulated transcription and impaired axonal transport (Millecamps and Julien, 2013). Increasing evidence shows that autophagy is implicated in neuronal disorders such as Alzheimer's disease (AD), Parkinson's disease (PD), Huntington's disease (HD), Amyotrophic lateral sclerosis (ALS), and Multiple sclerosis (MS). In this review, we will summarize and discuss the relationship between autophagy and these neurodegenerative disorders.

\section{AUTOPHAGY IN NEURODEGENERATION}

Regulation of autophagy in Alzheimer's disease (AD) $A D$ is the most common type of progressive dementia. In the early stages, the patient has difficulty in remembering recent events. As the disease advances, confusion, irritability, aggres- sion, mood swings, and trouble with reading and writing are displayed (Waldemar et al., 2007). AD is characterized by two abnormal structures, senile plaques and intra-neuronal fibrillary tangles. Amyloid plaques consist of largely insoluble toxic protein peptides, beta-amyloid peptides $(A \beta)$, which are generated by the enzymatic cleavage of a larger protein, amyloid precursor protein (APP) (Querfurth and LaFeria, 2010). Neurofibrillary tangles consist of highly phosphorylated forms of the microtubule-associated protein tau. Tau can interact with tubulin to stabilize microtubules and controls the vesicle transport in neurons. When this protein is abnormally hyperphosphorylated by tau kinases, tau is prone to aggregate into paired helical filaments forming neurofibrillary tangles (Wang et al., 2013).

The first evidence that autophagy may be involved in the pathogenesis of $A D$ came from the observation of numerous autophagic vacuoles (AVs) in $A D$ brains. Using immunogoldlabeling and electron microscopy, striking accumulation of immature AVs were observed in dystrophic neurites (Nixon et al., 2005). The purified AVs contained APP and beta-cleaved APP These vacuoles are highly enriched with presenilin-1 (PS1) and nicastrin together with $A \beta$. Along with this observation, additional evidence suggests that the transport of $A V s$ and autophagosome-lysosome fusion may be impaired in AD. Because the retrograde transport of autophagosomes along the axon is crucial for the fusion with lysosomes in neurons, the impaired retrograde transport of autophagosomes leads to the accumulation of immature $A V s$ and $A \beta$-generating $A V$ in $A D$ (UłamekKozioł et al., 2013; Yu et al., 2005), leading to enhanced $A \beta$ production. In addition, $A \beta$ was also shown to regulate autophagy by inducing the formation of AVs through AMPK activation (Son et al., 2012) and to induce autophagy through lipid raft-localized BECN1 (Nah et al., 2013).

There is an interesting report showing a gamma-secretaseindependent function of PS1 in lysosome. Although PS1 is a critical component of the APP-cleaving gamma-secretase complex, Lee et al. (2010) showed that PS1 mutations worsen lysosomal function and promote neuronal loss. In PS1 null blastocysts, substrate proteolysis and autophagosome clearance are inhibited as a result of lysosomal dysfunction, which is caused by the failure of PS1-dependent targeting of v-ATPase V0a1 subunit to lysosomes. Together with UPS, autophagy is known to play a role in the clearance of soluble monomeric and oligomeric tau and insoluble tau aggregates. In particular, inhibition of autophagosome-lysosome fusion by chloroquine causes a delay in tau clearance and the accumulation of tau aggregates (Hamano et al., 2008). Among the various forms of tau, phosphorylated tau is affected by the failure of autophagy (Rodríguez-Martín et al., 2013). In addition, truncated tau, which is found in AD brains (Gamblin et al., 2003), is cleared by autophagy in the tetracycline-inducible model, whereas fulllength tau is degraded by the proteasome (Dolan and Johnson, 2010). Furthermore, BECN1 downregulation is observed in early AD (Pickford et al., 2008) and is caused by caspasecleavage (Rohn et al., 2011). BECN1 reduction impairs retromer trafficking and phagocytosis in microglia (Lucin et al., 2013), affects APP processing in neurons (Jaeger et al., 2010), and increases $A \beta$ deposition and neurodegeneration in APP transgenic mouse, an AD model (Pickford et al., 2008). Evidence shows a pivotal role of autophagy in $A \beta$ accumulation and the formation of neurofibrillary tangles.

Because most neurodegenerative diseases are caused by abnormal protein aggregation, new discovery that can enhance the degradation of toxic aggregates is essential for effective therapy. Thus, autophagy, which is mainly responsible for the 
Table 1. Autophagy-enhancing compounds that therapeutically targets neurodegenerative diseases

\begin{tabular}{|c|c|c|c|c|}
\hline Disease & Autophagy target & Compound & Clinical trial phase & References \\
\hline \multirow[t]{9}{*}{$A D$} & TORC1 inhibition & Rapamycin & & Caccamo et al., 2010 \\
\hline & & SMER28 & & Tian et al., 2011 \\
\hline & & Metformin & Clinical trial phase II & Kickstein et al., 2010 \\
\hline & & Latrepirdine & Clinical trial phase III & Steele and Gandy, 2013 \\
\hline & & Resveratrol & Clinical trial phase III & Vingtdeux et al., 2010 \\
\hline & & RSVA314, 405 & & Vingtdeux et al., 2011 \\
\hline & AMPK activation & Lithium & Clinical trial phase II & Forlenza et al., 2012 \\
\hline & BECN1 complex & BECN1 mimetics & & Shoji-Kawata et al., 2013 \\
\hline & Iysosomal function & Nicotinamide & Clinical trial phase I & Liu et al., 2013 \\
\hline \multirow[t]{8}{*}{ PD } & TORC1 inhibition & Resveratrol & & Lin et al., 2014 \\
\hline & & Kaempferol & & Filomenin et al., 2012 \\
\hline & & Celastrol & & Deng et al., 2013 \\
\hline & & Curcumin & & Jiang et al., 2013 \\
\hline & BECN1 complex & BECN1 gene & & Spencer et al., 2009 \\
\hline & Vesicle transport & RAB1A gene & & Coune et al., 2011 \\
\hline & HDAC regulation & HDAC6 gene & & Du et al., 2010 \\
\hline & TFEB regulation & TFEB gene & & Decressac et al., 2013 \\
\hline \multirow[t]{6}{*}{ HD } & TORC1 inhibition & Rapamycin & & Ravikumar et al., 2004 \\
\hline & & $\mathrm{CCl}-779$ & & Ravikumar et al., 2004 \\
\hline & AMPK activation & Trehalose & & Tanaka et al., 2004 \\
\hline & & Rilmenidine & & Rose et al., 2010 \\
\hline & & $10-N S P$ & & Tsvetkov et al., 2010 \\
\hline & HDAC regulation & HDAC inhibitors & & Burli et al., 2013 \\
\hline \multirow[t]{3}{*}{ ALS } & TORC1 inhibition & Rapamycin & & Staats et al., 2013 \\
\hline & AMPK activation & Lithium & & Fornai et al., 2008 \\
\hline & & Trehalose & & Zhang et al., 2014 \\
\hline
\end{tabular}

degradation of abnormal proteins or organelles, is considered as an emerging therapeutic target. Moreover, autophagy plays a protective role against various stress stimuli and apoptotic insults (Giordano et al., 2014). Thus, the identification of autophagy inducers can be a good therapeutic strategy. Recently, non-toxic small-molecules that can restore basal-level of autophagy in neurons have been studied. Autophagy-inducing compounds show substantial therapeutic effects on various neurodegenerative disease animal models. Rapamycin, a selective inhibitor of TORC1, ameliorates $A \beta$ and tau pathology in an AD mouse model (Caccamo et al., 2010). Latrepirdine, also known as dimebon, stimulates Atg5-dependent autophagy in the mouse brain and reduces $A \beta$ neuropathology (Steele and Gandy, 2013). Protein phosphatase 2A (PP2A) agonist, metformin, inhibits tau hyperphosphorylation through inhibiting TORC1 and is currently tested in clinical trials for AD (Kickstein et al., 2010). In addition, SMER28, small-molecule enhancer of rapamycin 28 , greatly decreases the level of $A \beta$ peptide and APP-CTF in a gamma-secretase-independent manner (Tian et al., 2011).

Autophagy can also be induced by activating the ULK1 kinase, AMPK, in a TORC1-independent manner. Recently, various experimental and clinical trials have suggested that lithium may ameliorate $A D$ pathogenesis through a combination of mechanisms that includes AMPK activation and the regulation of autophagy (Forlenza et al., 2012). Resveratrol and its analogs, RSVA314 and RSVA405, have multiple molecular actions that include AMPK activation and show protective effect against $A D$ (Vingtdeux et al., 2010). Nicotinamide prevents pathology and cognitive decline through enhancing lysosome/autophagosome acidification to reduce autophagosome accumulation in an $A D$ mouse model (Liu et al., 2013). Even though the pathologic evidence is not sufficient in $A D$, virally packaged BECN1 and smallmolecule BECN1 mimetics can also reduce the accumulation of toxic aggregates by targeting the early stage of autophagy (Shoji-Kawata et al., 2013). It appears that, in the early stage of $A D$, autophagy-inducing agents can prevent the accumulation of plaques and tangles by degrading these aggregates. However, autophagy promotion can aggravate the pathologies in the late stage of $A D$ in which autophagosome/lysosome fusion or lysosomal function is impaired (Ching and Weihl, 2013). Therefore, new strategies that can enhance the fusion of autophagosome/lysosome and the lysosomal activity will be essential for advanced AD therapy.

Regulation of autophagy in Parkinson's disease (PD) $\mathrm{PD}$ is a chronic and progressive movement disorder. PD symptoms develop gradually over time. PD results from the death of vital nerve cells in the brain and primarily affects the substantia nigra. During PD development, the amounts of dopaminergic neurons are decreased in the substantia nigra (Tan et al., 2014). $\mathrm{PD}$ is well-characterized by the accumulation of $\alpha$-synuclein (SNCA) and ubiquitin into intracytoplasmic inclusions called Lewy bodies. Most PD cases are sporadic, meaning that they 
occur with unknown etiology and only $5 \%$ of PD cases are hereditary, due to mutations of at least 6 genes, SNCA, Parkin (PARK2), $\beta$-glucocerebrosidase (GBA), PTEN-induced putative kinase 1 (PINK1), DJ1, and leucine-rich repeat kinase 2 (LRRK2) (Alcalay et al., 2010).

The autophagic degeneration in melanized neurons of the substantia nigra in patients with PD provides evidence that autophagy is related to PD (Anglade et al., 1997). Emerging evidence then implicated that proteins encoded by PD-related genes can regulate autophagy pathways. Macroautophagy, chaperone-mediated autophagy (CMA), and mitophagy are involved in PD. SNCA level is a major determinant of its neurotoxicity and is related to the formation of Lewy bodies. Thus, SNCA degradation is essential in PD therapeutics. Recent studies indicate that deubiquitinated SNCA is mainly degraded by autophagy, while monoubiquitinated SNCA is preferentially removed by the proteasome (Pan and Yue, 2014). In SNCA transgenic mice, the ubiquitin-proteasome system is the main degradation pathway for SNCA under normal conditions, while autophagy is functional against increased intracellular SNCA (Ebrahimi-Fakhari et al., 2011). Mutations in the LRRK2 gene were first identified in 2004 in families with autosomal-dominant PD and G2019S mutation in LRRK2 is now found not only in 5$6 \%$ of familial PD, but also in $1-2 \%$ of patients with sporadic PD (Bonifati, 2006). Several reports indicated that LRRK2 might regulate macroautophagy. LRRK2 knockdown increases the autophagic flux under starvation conditions and inhibition of LRRK2 kinase activity stimulates macroautophagy in the absence of any alteration of TORC1 (Alegre-Abarrategui et al., 2009; Manzoni et al., 2013).

Mitophagy is also known to be linked with the function of several autosomal recessive PD-related genes. Wild-type Parkin, a cytosolic E3-like ligase, mainly localizes in the cytosol in the resting state and, following treatment with a mitochondrial uncoupler (CCCP), translocates to damaged mitochondria for clearance (Narendra et al., 2008). Thus, mutations in Parkin, which causes early-onset familial and sporadic PD, abolish the ability to clear damaged mitochondria through mitophagy. Further, studies revealed that depolarization of mitochondria triggers PINK1 accumulation on the outer membrane of damaged mitochondria to recruit Parkin. Parkin then labels some mitochondrial proteins such as VDAC and MFN with ubiquitin on the mitochondria, leading to the recruitment of several autophagy components, including p62, for the degradation of damaged mitochondria by mitophagy (Scarffe et al., 2014). However, despite extensive investigations, the direct connection between mitophagy and the cause of PD remains elusive.

Macroautophagy is not the only lysosomal degradation pathway responsible for SNCA degradation. Indeed, SNCA can be degraded through CMA. SNCA protein contains a KFERQ-like motif. Thus, SNCA is recognized by HSC70 chaperone and LAMP2A receptor for its degradation via the CMA pathway. Surprisingly, both pathogenic mutant forms of SNCA (A30P, A53T) and dopamine-modified SNCA can bind to LAMP2A, but fail to translocate to lysosomes (Martinez-Vicente et al., 2008). A recent study shows that LRRK2 can also be degraded in lysosomes by CMA, whereas the most common pathogenic mutant form of LRRK2 (G2019S) is rarely degraded by CMA (Orenstein et al., 2013).

In addition, oxidative stress, which is a key contributor in the loss of dopaminergic neurons, is known to cause PD. Thus, decreasing oxidative stress through autophagy can be an effective PD therapeutic strategy (Surendran and Rajasankar, 2010). Antioxidants such as thiols, ascorbic acid, and polyphenols, eliminate free radical intermediates and inhibit oxidation reaction. The ideal antioxidant for PD therapy should have both aberrant ROS clearing and autophagy-inducing properties because certain levels of oxidative stress are necessary to activate autophagy. Several polyphenols can be effective PD therapeutic agents because they can induce autophagy as well as reducing oxidative stress. For example, resveratrol partially protects rotenone-induced neurotoxicity in dopaminergic SHSY5Y cells through autophagy activation (Lin et al., 2014). Kaempferol and celastrol also prevent rotenone-mediated acute toxicity by increasing autophagy in SH-SY5Y cells (Filomeni et al., 2012; Deng et al., 2013). Moreover, curcumin, a diaryheptanoid possessing antioxidant properties, ameliorates the neurotoxicity in the A53T SNCA cell model of PD through recovery of macroautophagy (Jiang et al., 2013).

Because the basal level of autophagy is essentially required for maintaining neuronal homeostasis, enhancement of basal autophagy is explored as a therapeutic target. First of all, BECN1 gene transfer ameliorates the neurodegenerative pathology in the SNCA model of PD (Spencer et al., 2009). Overexpression of RAB1A, a key regulator of intracellular membrane trafficking and autophagosome assembly, was shown to partially improve motor deficits in SNCA-expressing dopaminergic neurons (Coune et al., 2011). Histone deacetylase 6 (HDAC6), which controls autophagosome maturation, is able to suppress SNCAinduced dopaminergic neuronal loss in drosophila (Du et al., 2010). In addition, transcription factor EB (TFEB) overexpression rescues dopamine neurons via autophagic clearance of SNCA oligomers (Decressac et al., 2013). At last, reduced levels of LAMP2A and HSC70 protein, the major components of CMA, have been observed in the brain of patients with PD. Thus, these findings provide a significant role of autophagy in the pathogenesis of PD. Autophagy represents a good target for PD therapeutics (Sala et al., 2014).

Regulation of autophagy in Huntington's disease (HD) $\mathrm{HD}$ is a neurodegenerative disorder that is characterized by the loss of motor control and cognitive dysfunction. HD is caused by damages to the subcortical part of the forebrain, the striatum, which plays a key role in body movements. HD is an autosomal dominant disease caused by the expansion of a CAG (cytosine-adenine-guanine) triplet repeat in exon 1 of the gene encoding huntingtin (HTT) protein. The polyglutamine (polyQ) tract in the mutant HTT protein encoded from the CAG repeat is responsible for the formation of toxic oligomers and aggregates. Whether these aggregates are toxic remain to be elucidated (Martin et al., 2015). The expansion of the HTT polyQ tract is 634 in normal individuals and $36-121$ in affected individuals. The longer the length of tandem repeats in mutant HTT is, the easier it is to form aggregates and the earlier is the age of HD onset (Juenemann et al., 2013). On the other hand, wild-type HTT function is not yet well-elucidated, but is essential for embryonic development (Duyao et al., 1995). Therefore, HD therapeutic approach should consider the importance of selectivity without interfering with wild-type HTT.

Mutant HTT forms perinuclear cytoplasmic aggregates and intracellular inclusions, which can be removed by autophagy. Previous studies have shown that autophagy induces the degradation of both aggregated and soluble forms of HTT and decreases toxicity in cell, fly, and mouse models of HD (Ravikumar et al., 2004). Moreover, autophagy alteration has been observed in various types of HD models such as primary striatal neurons from HD mice or lymphoblasts of patients with HD (Nagata et al., 2004). In general, inefficient macroautophagy is known to con- 


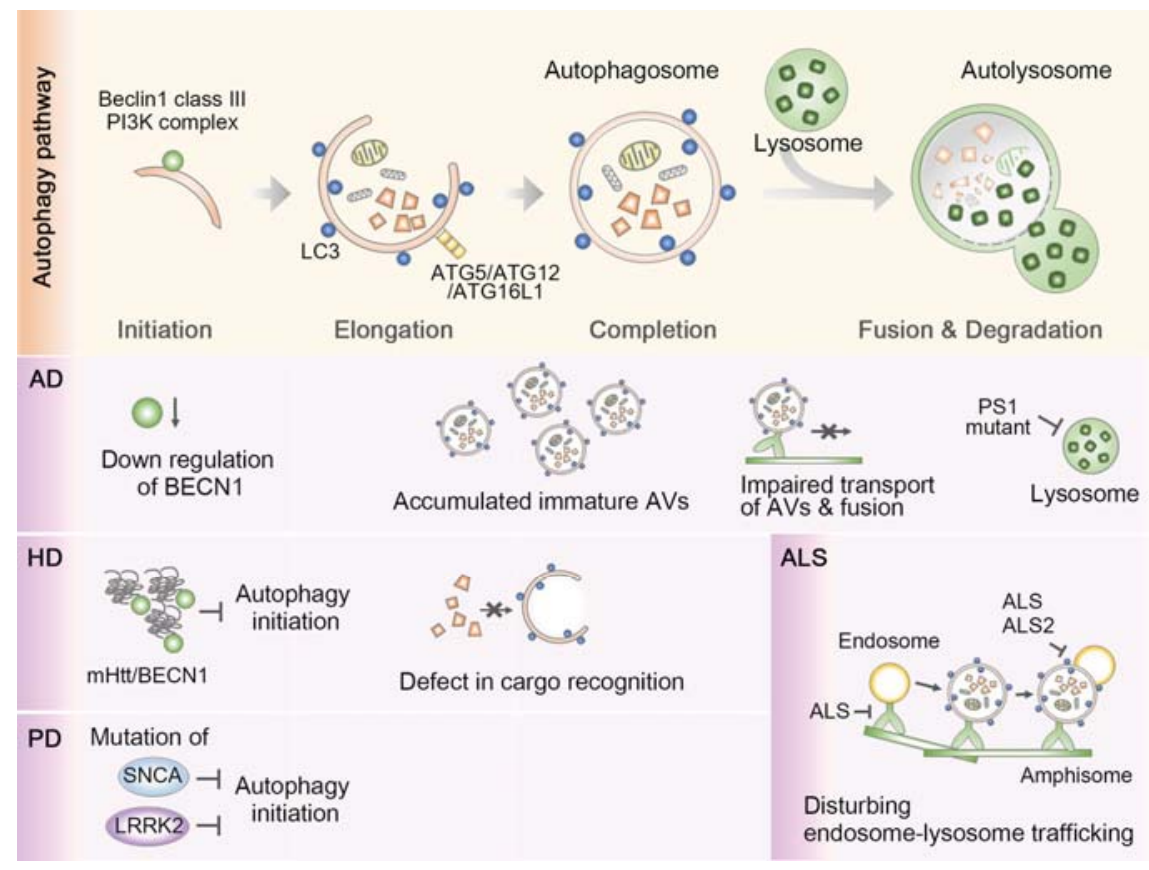

Fig. 1. Dysfunction of autophagy in neurodegeneration

tribute to HD pathogenesis. More specifically, the ability of AVs to recognize cytosolic cargos is largely defective in HD cells. The autophagosome-lysosome pathway is normal or even increased in HD cells, but AVs fail to efficiently recognize and trap cytosolic cargos due to the interaction between mutant HTT and p62 (Martinez-Vicente et al., 2010). In addition, the accumulated mutant HTT can recruit cytosolic BECN1 and impairs BECN1 complex-mediated autophagy, causing the accumulation of mutant HTT and leading to neuronal toxicity in patients with HD (Shibata et al., 2006).

Increasing evidence supports the fact that HTT is also degraded by CMA. While CMA activity increases in response to macroautophagy dysfunction in the early stages of $\mathrm{HD}$, this compensatory CMA activity continuously decreases with aging, leading to the onset of pathological symptoms (Koga et al., 2011). There is a KFERQ-like motif in the HTT fragment (1-552) (Qi and Zhang, 2014), raising the possibility that HTT can be affected by CMA. This notion is also in line with the observation that HSP70 and its co-chaperone, HSP40, modulates polyQ aggregation by partitioning monomeric conformations (Wacker et al., 2004). Accordingly, LAMP2A is involved in the chaperone/HTT clearance. A study showed that HTT is phosphorylated by $l_{\kappa} B$ kinase and the phosphorylated HTT can be better cleared by LAMP2A and HSP70-mediated CMA through additional post-translational modifications (Thompson et al., 2009).

HD pathogenesis is strongly influenced by neuronal autophagy dysfunction. However the molecular events, leading to autophagy dysfunction, remain elusive. Many therapeutic approaches to treat $\mathrm{HD}$ are under development to manipulate autophagy. Several reports suggest that autophagy activation alleviates behavioral motor abnormalities and neuropathology in HD model systems (Cortes and La Spada, 2014). Rapamycin and its analog $\mathrm{CCl}-779$ reduce polyQ toxicity and improve behavioral motor phenotypes in a HD mouse model (Ravikumar et al., 2004). Furthermore, mTOR-independent autophagy enhancer enhances HTT clearance and ameliorates HD motor phenotype. Treha- lose, a disaccharide present in many non-mammalian species, induces macroautophagy in an mTOR-independent manner and ameliorates polyQ-mediated pathology in a HD mouse model (Tanaka et al., 2004). Similarly, rilmenidine, an imidazoline-1 receptor agonist, induces mTOR-independent autophagy and reduces the pathologic signs of the disease in a HD mouse model (Rose et al., 2010). In addition, N(10)-substituted phenoxazine safely upregulates autophagy in an AKT- and mTOR-independent fashion in neurons. In a HD neuron model, N(10)-substituted phenoxazine is neuroprotective and decreases the accumulation of aggregated mutant HTT (Tsvetkov et al., 2010). Inhibition of histone deacetylase (HDAC) has also been considered as a therapeutic strategy for HD. HDAC6 was previously shown to be an essential link between autophagy activation and the UPS impairment to reduce neurodegeneration (Pandey et al., 2007). The HDAC1 and HDAC3 inhibitor, 4b, ameliorates cognitive decline and HD-related phenotypes in the striatum and cortex of R6/2 mice (Jia et al., 2012). Moreover, research is underway to identify potent and selective HDAC inhibitors as potential drugs for HD treatment (Bürli et al., 2013).

Regulation of autophagy in Amyotrophic lateral sclerosis (ALS)

ALS, often referred to as "Lou Gehrig's disease" is a motor neuron disease that is caused by a selective loss of upper and lower motor neurons in the brain and spinal cord. The progressive degeneration of motor neurons in ALS eventually leads to death due to respiratory failure. The most common form of ALS is a sporadic type and only about $5-10 \%$ of all ALS cases are hereditary. Since 1993, several genes associated with both familial ALS and sporadic ALS, have been identified, including superoxide dismutase 1 (SOD1), RNA-binding protein FUS, and TAR DNA-binding protein 43 (TDP43) (Andersen and AlChalabi, 2011). Many factors such as oxidative stress, mitochondrial dysfunction, abnormalities of the immune system, and glutamate toxicity are known to cause sporadic ALS (Kiernan et 


\section{al., 2011).}

Recently, dysfunction of the autophagic/lysosomal system was also shown to be tightly associated with ALS. The first evidence came from the SOD1G93A-expressing ALS mouse model. Although autophagy seems impaired in the ALS model, many researchers showed its induction in ALS mouse models (Morimoto et al., 2007; Song et al., 2012). In patients with sporadic ALS, the autophagy features were observed under electron microscopy in the cytoplasm of normal motor neurons and more frequently in degenerated motor neurons (Sasaki, 2011). However, this feature cannot ensure which factor causes the enhancement of LC3-II in ALS. Despite some controversy regarding the role of autophagy in ALS, many studies suggest that autophagic clearance of mutant SOD1 is beneficial to ALS (Li et al., 2008). Small heat shock protein B8 (HSPB8) decreases the aggregation of mutant SOD1 and increases its solubility and clearance by enhancing autophagy without affecting wild-type SOD1 turnover in the SOD1G93A ALS model mice (Crippa et al., 2010). A dramatic decrease in mutant SOD1 toxicity was also observed in X-box-binding protein-1 (XBP-1), a key molecule in unfolded protein response, deficient mice, correlating with the increased levels of autophagy and the reduced accumulation of mutant SOD1 aggregates in the spinal cord (Hetz et al., 2009). Furthermore, autophagy-linked FYVE (Alfy) promotes the autophagic degradation of misfolded proteins involved in ALS. In this study, Alfy overexpression decreases the expression of mutant proteins through autophagy and reduces mutant protein toxicity (Han et al., 2014). On the other hand, mutant SOD1 transgenic mice haploinsufficient for BECN1 show an unexpected increase in lifespan (Nassif et al., 2014), showing a pathogenic and opposite role of BECN1 in the development of ALS.

Additionally, functions of several ALS-related genes were reported to be associated with autophagy. ALS2/alsin is a guanine nucleotide exchange factor for the small GTPase Rab5 and is involved in micropinocytosis-associated endosome fusion and trafficking. Recently, ALS2 loss has been shown to exacerbate SOD1H46R-mediated neurotoxicity by disturbing endosome-autophagosome trafficking (Hadano et al., 2010). Another protein mutation causing ALS is TDP43, a nuclear RNA-binding protein involved in several aspects of RNA processing. TDP43 turnover is known to be enhanced by autophagy activation and that autophagy-activating compounds improve TDP43 clearance and enhance survival in neuronal ALS models (Barmada et al., 2014).

Unlike other neurodegenerative disorders, the identification of autophagy-regulating drugs as potential ALS therapeutic agents is not much studied. Like other diseases, however, autophagic degradation of mutant SOD1 and TDP43 is believed to be beneficial to ALS. Therefore, several autophagic enhancers such as rapamycin, lithium, and trehalose are expected to function as pathology reliever in ALS. In general, rapamycin plays a neuroprotective role in several neurodegenerations. However, in ALS models, it showed beneficial and detrimental effects in various studies. Rapamycin accelerates motor neuron degeneration and shortens the lifespan of SOD1G93A mice (Zhang et al., 2011). In contrast, rapamycin moderately increases the survival of ALS mice deficient of mature lymphocytes. (Staats et al., 2013). In a recent study, mTOR-independent autophagy enhancer, trehalose, decreased SOD1 and p62 aggregation, reduced ubiquitinated protein accumulation, and inhibited the proapoptotic pathway in SOD1G93A mice (Zhang et al., 2014). Lithium also shows both protective and detrimental effects in ALS models. Lithium delay disease progression in patients with ALS. Moreover, it showed neuroprotective effects and delayed the disease onset and duration in the SOD1G93A ALS mouse model (Fornai et al., 2008). In contrast, under the same conditions, another group showed that lithium does not ameliorate disease progression in SOD1G93A mice (C57BL/6J or 129S2/Sv strains) (Pizzasegola et al., 2009). While this aspect needs to be further characterized using animal genetic models, combination strategies or modified autophagy enhancers may still be appropriate as ALS therapeutic approaches.

\section{CONCLUSION}

Accumulated evidence revealed that neuronal autophagy is essential for the healthy aging of neurons. Moreover, neuronal autophagy is the major process for the degradation of an abnormal protein aggregate, which is the major cause of most neurodegenerative diseases such as $A D, P D, H D$, and ALS. Increasing research in autophagy revealed several links connecting autophagy and neurodegenerative diseases. However, direct links and molecular mechanisms remain elusive and need to be further addressed. Impairment of lysosomal function or autophagosome/lysosome fusion is observed in most neurodegenerative disorders. Nevertheless, the recent attempts to treat the autophagic impairment in neurodegeneration have focused on the induction of initial autophagy. Therefore, it is critical to overcome lysosomal dysfunction when developing therapeutic strategies against neurodegenerative diseases. Research on autophagy as a potential therapeutic target for neurodegenerative disease treatment is only starting. Some compounds for the treatment of AD have been tested in human clinical trials. Other compounds for the treatment of the other neurodegenerative diseases are now in the preclinical phase. Despite research limitations, therapeutic approaches targeting autophagy are highly expected to contribute to the treatment of neurodegenerative diseases.

\section{ACKNOWLEDGMENTS}

This work was supported by the grants from the Global Research Laboratory Program (GRL, NRF-2010-00341) and the CRI grant (NRF 2013RIA2A1A01016896), funded by the Ministry of Education, Science and Technology (MEST) in Korea.

\section{REFERENCES}

Abrahamsen, H., Stenmark, H., and Platta, H.W. (2012). Ubiquitination and phosphorylation of Beclin 1 and its binding partners: Tuning class III phosphatidylinositol 3-kinase activity and tumor suppression. FEBS Lett. 586, 1584-1591.

Alcalay, R.N., Caccappolo, E., Mejia-Santana, H., Tang, M.X., Rosado, L., Ross, B.M., Verbitsky, M., Kisselev, S., Louis, E.D. Comella, C., et al. (2010). Frequency of known mutations in early-onset Parkinson disease: implication for genetic counseling the consortium on risk for early onset Parkinson disease study. Arch. Neurol. 67, 1116-1122.

Alegre-Abarrategui, J., Christian, H., Lufino, M.M., Mutihac, R., Venda, L.L., Ansorge, O., and Wade-Martins, R. (2009). LRRK2 regulates autophagic activity and localizes to specific membrane microdomains in a novel human genomic reporter cellular model. Hum. Mol. Genet. 18, 4022-4034.

Andersen, P.M., and Al-Chalabi, A. (2011). Clinical genetics of amyotrophic lateral sclerosis: what do we really know? Nat. Rev. Neurol. 7, 603-615.

Anglade, P., Vyas, S., Javoy-Agid, F., Herrero, M.T., Michel, P.P., Marquez, J., Mouatt-Prigent, A., Ruberg, M., Hirsch, E.C., and Agid, Y. (1997). Apoptosis and autophagy in nigral neurons of patients with Parkinson's disease. Histol. Histopathol. 12, 25-31. 
Barmada, S.J., Serio, A., Arjun, A., Bilican, B., Daub, A., Ando, D.M., Tsvetkov, A., Pleiss, M., Li, X., Peisach, D., et al. (2014). Autophagy induction enhances TDP43 turnover and survival in neuronal ALS models. Nat. Chem. Biol. 10, 677-685.

Bonifati, V. (2006). Parkinson's disease: the LRRK2-G2019S mutation: opening a novel era in Parkinson's disease genetics. Eur. J. Hum. Genet. 14, 1061-1062.

Boya, P., Reggiori, F., and Codogno, P. (2013). Emerging regulation and functions of autophagy. Nat. Cell Biol. 15, 713-720.

Bürli, R.W., Luckhurst, C.A., Aziz, O., Matthews, K.L., Yates, D., Lyons, K.A., Beconi, M., McAllister, G., Breccia, P., Stott, A.J., et al. (2013). Design, synthesis, and biological evaluation of potent and selective class lla histone deacetylase (HDAC) inhibitors as a potential therapy for Huntington's disease. J. Med. Chem. 56, 9934-9954.

Caccamo, A., Majumder, S., Richardson, A., Strong, R., and Oddo, S. (2010). Molecular interplay between mammalian target of rapamycin (mTOR), amyloid-beta, and Tau: effects on cognitive impairments. J. Biol. Chem. 285, 13107-13120.

Chen, D., Fan, W., Lu, Y., Ding, X., Chen, S., and Zhong, Q. (2012) A mammalian autophagosome maturation mechanism mediated by TECPR 1 and the Atg12-Atg5 conjugate. Mol. Cell. 45, 629641.

Cherra, S.J. 3rd, and Chu, C.T. (2008). Autophagy in neuroprotection and neurodegeneration: A question of balance. Future Neurol. 3, 309-323.

Ching, J.K., and Weihl, C.C. (2013). Rapamycin-induced autophagy aggravates pathology and weakness in a mouse model of VCPassociated myopathy. Autophagy 9, 799-800.

Cortes, C.J, and La Spada, A.R. (2014). The many faces of autophagy dysfunction in Huntington's disease: from mechanism to therapy. Drug Discov. Today 19, 963-971.

Coune, P.G., Bensadoun, J.C., Aebischer, P., and Schneider, B.L. (2011). Rab1A over-expression prevents Golgi apparatus fragmentation and partially corrects motor deficits in an alphasynuclein based rat model of Parkinson's disease. J. Parkinsons Dis. 1, 373-387.

Crippa, V., Sau, D., Rusmini, P., Boncoraglio, A., Onesto, E., Bolzoni, E., Galbiati, M., Fontana ,E., Marino, M., Carra, S., et al. (2010). The small heat shock protein B8 (HspB8) promotes autophagic removal of misfolded proteins involved in amyotrophic lateral sclerosis (ALS). Hum. Mol. Genet. 19, 3440-3456.

Decressac, M., Mattsson, B., Weikop, P., Lundblad, M., Jakobsson, J., and Björklund, A. (2013). TFEB-mediated autophagy rescues midbrain dopamine neurons from $\alpha$-synuclein toxicity. Proc. Natl. Acad. Sci. USA 110, E1817-1826.

Deng, Y.N., Shi, J., Liu, J., and Qu, Q.M. (2013). Celastrol protects human neuroblastoma SH-SY5Y cells from rotenone-induced injury through induction of autophagy. Neurochem. Int. 63, 1-9.

Dolan, P.J., and Johnson, G.V. (2010). A caspase cleaved form of tau is preferentially degraded through the autophagy pathway. J. Biol. Chem. 285, 21978-21987.

Du, G., Liu, X., Chen, X., Song, M., Yan, Y., Jiao, R., and Wang, C.C. (2010). Drosophila histone deacetylase 6 protects dopaminergic neurons against \{alpha\}-synuclein toxicity by promoting inclusion formation. Mol. Biol. Cell 21, 2128-2137.

Duyao, M.P., Auerbach, A.B., Ryan, A., Persichetti, F., Barnes, G.T., McNeil, S.M., Ge, P., Vonsattel, J.P., Gusella, J.F., Joyner, A.L., et al. (1995). Inactivation of the mouse Huntington's disease gene homolog Hdh. Science 269, 407-410.

Ebrahimi-Fakhari, D., Cantuti-Castelvetri, I., Fan, Z., Rockenstein, E. Masliah, E., Hyman, B.T., McLean, P.J., and Unni, V.K (2011). Distinct roles in vivo for the ubiquitin-proteasome system and the autophagy-lysosomal pathway in the degradation of $\alpha$ synuclein. J. Neurosci. 31, 14508-14520.

Filimonenko, M., Isakson, P., Finley, K.D., Anderson, M., Jeong, H., Melia, T.J., Bartlett, B.J., Myers, K.M., Birkeland, H.C., Lamark, T. et al. (2010). The selective macroautophagic degradation of aggregated proteins requires the PI3P-binding protein Alfy. Mol. Cell 38, 265-279.

Filomeni, G., Graziani, I., De Zio, D., Dini, L., Centonze, D., Rotilio G., and Ciriolo, M.R. (2012). Neuroprotection of kaempferol by autophagy in models of rotenone-mediated acute toxicity: possible implications for Parkinson's disease. Neurobiol. Aging 33, 767-785.

Forlenza, O.V., de Paula, V.J., Machado-Vieira, R., Diniz, B.S., and
Gattaz, W.F. (2012). Does lithium prevent Alzheimer's disease? Drugs Aging 29, 335-342.

Fornai, F., Longone, P., Cafaro, L., Kastsiuchenka, O., Ferrucci, M., Manca, M.L., Lazzeri, G., Spalloni, A., Bellio, N., Lenzi, P., et al (2008). Lithium delays progression of amyotrophic lateral sclerosis. Proc. Natl. Acad. Sci. USA 105, 2052-2057.

Gamblin, T.C., Chen, F., Zambrano, A., Abraha, A., Lagalwar, S., Guillozet, A.L., Lu, M., Fu, Y., Garcia-Sierra, F., LaPointe, N., et al. (2003). Caspase cleavage of tau: linking amyloid and neurofibrillary tangles in Alzheimer's disease. Proc. Natl. Acad. Sci. USA 100, 10032-10037.

Giordano, S., Darley-Usmar, V., and Zhang, J. (2014). Autophagy as an essential cellular antioxidant pathway in neurodegenerative disease. Redox Biol. 2, 82-90.

Hadano, S., Otomo, A., Kunita, R., Suzuki-Utsunomiya, K., Akatsuka A., Koike, M., Aoki, M., Uchiyama, Y., Itoyama, Y., and Ikeda, J.E. (2010). Loss of ALS2/Alsin exacerbates motor dysfunction in a SOD1-expressing mouse ALS model by disturbing endolysosomal trafficking. PLoS One 5, e9805.

Hamano, T., Gendron, T.F., Causevic, E., Yen, S.H., Lin, W.L., Isidoro, C., Deture, M., and Ko, L.W. (2008). Autophagic-lysosomal perturbation enhances tau aggregation in transfectants with induced wild-type tau expression. Eur. J. Neurosci. 27, 1119-1130.

Han, H., Wei, W., Duan, W., Guo, Y., Li, Y., Wang, J., Bi, Y., and Li, C. (2014). Autophagy-linked FYVE protein (Alfy) promotes autophagic removal of misfolded proteins involved in amyotrophic lateral sclerosis (ALS). In Vitro Cell. Dev. Biol. Anim. [Epub ahead of print]

Hetz, C., Thielen, P., Matus, S., Nassif, M., Court, F., Kiffin, R., Martinez, G., Cuervo, A.M., Brown, R.H., and Glimcher, L.H. (2009). XBP-1 deficiency in the nervous system protects against amyotrophic lateral sclerosis by increasing autophagy. Genes Dev. 23, 2294-2306.

Hyttinen, J.M., Niittykoski, M., Salminen, A., and Kaarniranta, K. (2013). Maturation of autophagosomes and endosomes: a key role for Rab7. Biochim. Biophys. Acta 1833, 503-510.

Jaeger, P.A., Pickford, F., Sun, C.H., Lucin, K.M., Masliah, E., and Wyss-Coray, T. (2010). Regulation of amyloid precursor protein processing by the Beclin 1 complex. PLoS One 5, e11102.

Jia, H., Kast, R.J., Steffan, J.S and Thomas, E.A. (2012). Selective histone deacetylase (HDAC) inhibition imparts beneficial effects in Huntington's disease mice: implications for the ubiquitinproteasomal and autophagy systems. Hum. Mol. Genet. 21, 5280-5293.

Jiang, T.F., Zhang, Y.J., Zhou, H.Y., Wang, H.M., Tian, L.P., Liu, J., Ding, J.Q., and Chen, S.D. (2013). Curcumin ameliorates the neurodegenerative pathology in A53T $\alpha$-synuclein cell model of Parkinson's disease through the downregulation of mTOR/ p70S6K signaling and the recovery of macroautophagy. J. Neuroimmune Pharmacol. 8, 356-669.

Juenemann, K., Schipper-Krom, S., Wiemhoefer, A., Kloss, A., Sanz Sanz, A., and Reits, E.A. (2013). Expanded polyglutaminecontaining $\mathrm{N}$-terminal huntingtin fragments are entirely degraded by mammalian proteasomes. J. Biol. Chem. 288, 27068-27084.

Kaushik, S., and Cuervo, A.M. (2012). Chaperone-mediated autophagy: a unique way to enter the lysosome world. Trends Cell Biol. 22, 407-417.

Kesidou, E., Lagoudaki, R., Touloumi, O., Poulatsidou, K.N., and Simeonidou, C. (2013). Autophagy and neurodegenerative disorders. Neural Regen. Res. 8, 2275-2283.

Kickstein, E., Krauss, S., Thornhill, P., Rutschow, D., Zeller, R., Sharkey, J., Williamson, R., Fuchs, M., Köhler, A., Glossmann, $\mathrm{H}$., et al. (2010). Biguanide metformin acts on tau phosphorylation via mTOR/protein phosphatase 2A (PP2A) signaling. Proc Natl. Acad. Sci. USA 107, 21830-21835.

Kiernan, M.C., Vucic, S., Cheah, B.C., Turner, M.R., Eisen, A., Hardiman, O., Burrell, J.R., and Zoing, M.C. (2011). Amyotrophic lateral sclerosis. Lancet 377, 942-955.

Koga, H., Martinez-Vicente, M., Arias, E., Kaushik, S., Sulzer, D., and Cuervo, A.M. (2011). Constitutive upregulation of chaperone-mediated autophagy in Huntington's disease. J. Neurosci. 31, 18492-18505

Komatsu, M., and Ichimura, Y. (2010). Selective autophagy regulates various cellular functions. Genes Cells 15, 923-933.

Lee, J.A. (2012). Neuronal autophagy: a housekeeper or a fighter in neuronal cell survival? Exp. Neurobiol. 21,1-8. 
Lee, J.H., Yu, W.H., Kumar, A., Lee, S., Mohan, P.S., Peterhoff, C.M., Wolfe, D.M., Martinez-Vicente, M., Massey, A.C., Sovak, G., et al. (2010). Lysosomal proteolysis and autophagy require presenilin 1 and are disrupted by Alzheimer-related PS1 mutations. Cell 141, 1146-1158.

Li, L., Zhang, X., and Le, W. (2008). Altered macroautophagy in the spinal cord of SOD1 mutant mice. Autophagy 4, 290-293.

Li, W.W., Li, J., and Bao, J.K. (2012). Microautophagy: lesserknown self-eating. Cell. Mol. Life Sci. 69, 1125-1136.

Lin, T.K., Chen, S.D., Chuang, Y.C., Lin, H.Y., Huang, C.R., Chuang, J.H., Wang, P.W., Huang, S.T., Tiao, M.M., Chen, J.B., et al. (2014). Resveratrol partially prevents rotenone-induced neurotoxicity in dopaminergic SH-SY5Y cells through induction of heme oxygenase-1 dependent autophagy. Int. J. Mol. Sci. 15 1625-1646.

Liu, D., Pitta, M., Jiang, H., Lee, J.H., Zhang, G., Chen, X., Kawamoto, E.M., and Mattson, M.P. (2013). Nicotinamide forestalls pathology and cognitive decline in Alzheimer mice: evidence for improved neuronal bioenergetics and autophagy procession. Neurobiol. Aging 34, 1564-1580.

Lucin, K.M., O'Brien, C.E., Bieri, G., Czirr, E., Mosher, .KI., Abbey, R.J., Mastroeni, D.F., Rogers, J., Spencer, B., Masliah, E., et al (2013). Microglial beclin 1 regulates retromer trafficking and phagocytosis and is impaired in Alzheimer's disease. Neuron 79 873-886.

Manzoni, C., Mamais, A., Dihanich, S., Abeti, R., Soutar, M.P., PlunFavreau, H., Giunti, P., Tooze, S.A., Bandopadhyay, R., and Lewis, P.A. (2013). Inhibition of LRRK2 kinase activity stimulates macroautophagy. Biochim. Biophys. Acta 1833, 2900-2910.

Martin, D.D., Ladha, S., Ehrnhoefer, D.E., and Hayden, M.R. (2015). Autophagy in Huntington disease and huntingtin in autophagy. Trends Neurosci. 38, 26-35.

Martinez-Vicente, M., Talloczy, Z., Kaushik, S., Massey, A.C., Mazzulli, J., Mosharov, E.V., Hodara, R., Fredenburg R., Wu, D.C. Follenzi, A., et al. (2008). Dopamine-modified alpha-synuclein blocks chaperone-mediated autophagy. J. Clin. Invest. 118, 777 788.

Martinez-Vicente, M., Talloczy, Z., Wong, E., Tang, G., Koga, H., Kaushik, S., de Vries, R., Arias, E., Harris, S., Sulzer, D., et al. (2010). Cargo recognition failure is responsible for inefficient autophagy in Huntington's disease. Nat. Neurosci. 13, 567-576.

Millecamps, S., and Julien, J.P. (2013). Axonal transport deficits and neurodegenerative diseases. Nat. Rev. Neurosci. 14, 161-176.

Mizushima, N., Yoshimori, T., and Ohsumi, Y. (2011). The role of Atg proteins in autophagosome formation. Annu. Rev. Cell Dev. Biol. $27,107-132$

Mizushima, N. (2010). The role of the Atg1/ULK1 complex in autophagy regulation. Curr. Opin. Cell Biol. 22, 132-139.

Morimoto, N., Nagai, M., Ohta, Y., Miyazaki, K., Kurata, T., Morimoto, M., Murakami, T., Takehisa, Y., Ikeda, Y., Kamiya, T., et al. (2007). Increased autophagy in transgenic mice with a G93A mutant SOD1 gene. Brain Res. 1167, 112-117.

Nagata, E., Sawa, A., Ross, C.A., and Snyder, SH. (2004). Autophagosome-like vacuole formation in Huntington's disease lymphoblasts. Neuroreport 15, 1325-1328.

Nah, J., Pyo, J.O., Jung, S., Yoo, S.M., Kam, T.I., Chang, J., Han, J. Soo A An, S., Onodera, T., and Jung, Y.K. (2013). BECN1/Beclin 1 is recruited into lipid rafts by prion to activate autophagy in response to amyloid $\beta$ 42. Autophagy 9, 2009-2021.

Nair, U., Jotwani, A., Geng, J., Gammoh, N., Richerson, D., Yen, W.L., Griffith, J., Nag, S., Wang, K., Moss, T., et al. (2011). SNARE proteins are required for macroautophagy. Cell 146, 290-302.

Nakatogawa, H., Suzuki, K., Kamada, Y., and Ohsumi, Y. (2009). Dynamics and diversity in autophagy mechanisms: lessons from yeast. Nat. Rev. Mol. Cell Biol. 10, 458-467.

Narendra, D., Tanaka, A., Suen, D.F., and Youle, R.J. (2008). Parkin is recruited selectively to impaired mitochondria and promotes their autophagy. J. Cell Biol. 183, 795-803.

Nassif, M., Valenzuela, V., Rojas-Rivera, D., Vidal, R., Matus, S., Castillo, K., Fuentealba, Y., Kroemer, G., Levine, B., and Hetz, C. (2014). Pathogenic role of BECN1/Beclin 1 in the development of amyotrophic lateral sclerosis. Autophagy 10, 1256-1271.

Nixon, R.A., Wegiel, J., Kumar, A., Yu, W.H., Peterhoff, C., Cataldo, A., and Cuervo, A.M. (2005). Extensive involvement of autophagy in Alzheimer disease: an immuno-electron microscopy study. J. Neuropathol. Exp. Neurol. 64, 113-122.
Orenstein, S.J., Kuo, S.H., Tasset, I., Arias, E., Koga, H., Fernandez-Carasa, I., Cortes, E., Honig, L.S., Dauer, W., Consiglio, A. et al. (2013). Interplay of LRRK2 with chaperone-mediated autophagy. Nat. Neurosci. 16, 394-406.

Pan, P.Y., and Yue, Z. (2014). Genetic causes of Parkinson's disease and their links to autophagy regulation. Parkinsonism Relat. Disord. 20 Suppl 1, S154-157.

Pandey, U.B., Nie, Z., Batlevi, Y., McCray, B.A., Ritson, G.P., Nedelsky, N.B., Schwart, S.L., DiProspero, N.A., Knight, M.A., Schuldiner, O., et al. (2007). HDAC6 rescues neurodegeneration and provides an essential link between autophagy and the UPS. Nature 447, 859863.

Pickford, F., Masliah, E., Britschgi, M., Lucin, K., Narasimhan, R., Jaeger, P.A., Small, S., Spencer, B., Rockenstein, E., Levine, B., et al. (2008). The autophagy-related protein beclin 1 shows reduced expression in early Alzheimer disease and regulates amyloid beta accumulation in mice. J. Clin. Invest. 118, 2190 2199.

Pizzasegola, C., Caron, I., Daleno, C., Ronchi, A., Minoia, C., Carrì, M.T., and Bendotti, C. (2009). Treatment with lithium carbonate does not improve disease progression in two different strains of SOD1 mutant mice. Amyotroph. Lateral Scler. 10, 221-228.

Qi, L., and Zhang, X.D. (2014). Role of chaperone-mediated autophagy in degrading Huntington's disease-associated huntingtin protein. Acta Biochim. Biophys. Sin. (Shanghai) 46, 8391.

Querfurth, H.W., and LaFerla, F.M. (2010). Alzheimer's disease. N. Engl. J. Med. 362, 329-344.

Ravikumar, B., Vacher, C., Berger, Z., Davies, J.E., Luo, S., Oroz, L.G., Scaravilli, F., Easton, D.F., Duden, R., O'Kane, C.J., et al. (2004). Inhibition of mTOR induces autophagy and reduces toxicity of polyglutamine expansions in fly and mouse models of Huntington disease. Nat. Genet. 36, 585-595.

Rodríguez-Martín, T., Cuchillo-lbáñez, I., Noble, W., Nyenya, F., Anderton, B.H., and Hanger, D.P. (2013). Tau phosphorylation affects its axonal transport and degradation. Neurobiol. Aging 34, 2146-2157.

Rohn, T.T., Wirawan, E., Brown, R.J., Harris, J.R., Masliah, E., and Vandenabeele, P. (2011). Depletion of Beclin-1 due to proteolytic cleavage by caspases in the Alzheimer's disease brain. Neurobiol. Dis. $43,68-78$

Rose, C., Menzies, F.M., Renna, M., Acevedo-Arozena, A., Corrochano, S., Sadiq, O., Brown, S.D., and Rubinsztein, D.C. (2010) Rilmenidine attenuates toxicity of polyglutamine expansions in a mouse model of Huntington's disease. Hum. Mol. Genet. 19, 2144-2153.

Russell, R.C., Tian, Y., Yuan, H., Park, H.W., Chang, Y.Y., Kim, J., Kim, H., Neufeld, T.P., Dillin, A., and Guan, K.L. (2013). ULK1 induces autophagy by phosphorylating Beclin-1 and activating VPS34 lipid kinase. Nat. Cell Biol. 15, 741-750.

Sala, G., Stefanoni, G., Arosio, A., Riva, C., Melchionda, L., Saracchi, E., Fermi, S., Brighina, L., and Ferrarese, C. (2014). Reduced expression of the chaperone-mediated autophagy carrier hsc70 protein in lymphomonocytes of patients with Parkinson's disease. Brain Res. 1546, 46-52.

Sasaki, S. (2011). Autophagy in spinal cord motor neurons in sporadic amyotrophic lateral sclerosis. J. Neuropathol. Exp. Neurol. 70, 349-359.

Scarffe, L.A., Stevens, D.A., Dawson, V.L., and Dawson, T.M. (2014). Parkin and PINK1: much more than mitophagy. Trends Neurosci. 37, 315-324.

Shibata, M., Lu, T., Furuya, T., Degterev, A., Mizushima, N., Yoshimori, T., MacDonald, M., Yankner, B., and Yuan, J. (2006). Regulation of intracellular accumulation of mutant Huntingtin by Beclin 1. J. Biol. Chem. 281, 14474-14485.

Shibutani, S.T., and Yoshimori, T. (2014) A current perspective of autophagosome biogenesis. Cell Res. 24, 58-68.

Shintani, T., and Klionsky, D.J. (2004). utophagy in health and disease: a double-edged sword. Science 306, 990-995.

Shoji-Kawata, S., Sumpter, R., Leveno, M., Campbell, G.R., Zou, Z., Kinch, L., Wilkins, A.D., Sun, Q., Pallauf, K., MacDuff, D., et al. (2013). Identification of a candidate therapeutic autophagyinducing peptide. Nature 494, 201-206.

Shpilka, T., Mizushima, N., and Elazar, Z. (2012). Ubiquitin-like proteins and autophagy at a glance. J. Cell Sci. 125, 2343-2348

Son, S.M., Jung, E.S., Shin, H.J., Byun, J., and Mook-Jung, I. (2012) 
A $\beta$-induced formation of autophagosomes is mediated by RAGECaMKKB-AMPK signaling. Neurobiol. Aging 33, 1006.e11-23.

Song, C.Y., Guo, J.F., Liu, Y., and Tang, B.S. (2012). Autophagy and Its Comprehensive Impact on ALS. Int. J. Neurosci. 122, 695703.

Spencer, B., Potkar, R., Trejo, M., Rockenstein, E., Patrick, C., Gindi, R., Adame, A., Wyss-Coray, T., and Masliah, E. (2009). Beclin 1 gene transfer activates autophagy and ameliorates the neurodegenerative pathology in alpha-synuclein models of Parkinson's and Lewy body diseases. J. Neurosci. 29, 13578-13588.

Staats, K.A., Hernandez, S., Schönefeldt, S., Bento-Abreu, A., Dooley, J., Van Damme P., Liston, A., Robberecht, W., and Van Den Bosch, L. (2013). Rapamycin increases survival in ALS mice lacking mature lymphocytes. Mol. Neurodegener 8, 31.

Steele, J.W., and Gandy, S. (2013). Latrepirdine (Dimebon®), a potential Alzheimer therapeutic, regulates autophagy and neuropathology in an Alzheimer mouse model. Autophagy 9, 617618.

Surendran, S., and Rajasankar, S. (2010). Parkinson's disease: oxidative stress and therapeutic approaches. Neurol. Sci. 31, 531-540.

Tan, C.C., Yu, J.T., Tan, M.S., Jiang, T., Zhu, X.C., and Tan, L. (2014). Autophagy in aging and neurodegenerative diseases: implications for pathogenesis and therapy. Neurobiol. Aging 35, 941-957.

Tanaka, M., Machida, Y., Niu, S., Ikeda, T., Jana, N.R., Doi, H., Kurosawa, M., Nekooki, M., and Nukina, N. (2004). Trehalose alleviates polyglutamine-mediated pathology in a mouse model of Huntington disease. Nat. Med. 10, 148-154.

Thompson, L,M., Aiken, C,T., Kaltenbach, L.S., Agrawal, N., Illes, K., Khoshnan, A., Martinez-Vincente, M., Arrasate, M., O'Rourke, J.G., Khashwii, H., et al. (2009). IKK phosphorylates Huntingtin and targets it for degradation by the proteasome and lysosome. J. Cell Biol. 187, 1083-1099.

Tian, Y., Bustos, V., Flajolet, M., and Greengard, P. (2011). A smallmolecule enhancer of autophagy decreases levels of Abeta and APP-CTF via Atg5-dependent autophagy pathway. FASEB J. 25, 1934-1942.

Tsvetkov, A.S., Miller, J., Arrasate, M., Wong, J.S., Pleiss, M.A., and Finkbeiner, S. (2010). A small-molecule scaffold induces autophagy in primary neurons and protects against toxicity in a
Huntington disease model. Proc. Natl. Acad. Sci. USA. 107, 16982-16987.

Ułamek-Kozioł, M., Furmaga-Jabłońska, W., Januszewski, S., Brzozowska, J., Sciślewska, M., Jabłoński, M., and Pluta, R. (2013). Neuronal autophagy: self-eating or self-cannibalism in Alzheimer's disease. Neurochem. Res. 38, 1769-1773.

Vingtdeux, V., Giliberto, L., Zhao, H., Chandakkar, P., Wu, Q., Simon, J.E., Janle, E.M., Lobo, J., Ferruzzi. M.G., Davies. P., et al. (2010). AMP-activated protein kinase signaling activation by resveratrol modulates amyloid-beta peptide metabolism. J. Biol. Chem. 285, 9100-9113.

Wacker, J.L., Zareie, M.H., Fong, H., Sarikaya, M., and Muchowski P.J. (2004). Hsp70 and Hsp40 attenuate formation of spherical and annular polyglutamine oligomers by partitioning monomer. Nat. Struct. Mol. Biol. 11, 1215-1222.

Waldemar, G., Dubois, B., Emre, M., Georges, J., McKeith, I.G Rossor, M., Scheltens, P., Tariska, P., and Winblad, B. (2007). Recommendations for the diagnosis and management of Alzheimer's disease and other disorders associated with dementia: EFNS guideline. Eur. J. Neurol. 14, e1-26.

Wang, J.Z., Xia, Y.Y., Grundke-lqbal, I., and Iqbal, K. (2013). Abnormal hyperphosphorylation of tau: sites, regulation, and molecular mechanism of neurofibrillary degeneration. J. Alzheimers Dis. 33 Suppl 1, S123-139.

Weidberg, H., Shvets, E., and Elazar, Z. (2011). Biogenesis and cargo selectivity of autophagosomes. Annu. Rev. Biochem. 80, 125-156.

Yu, W.H., Cuervo, A.M., Kumar, A., Peterhoff, C.M., Schmidt, S.D., Lee, J.H., Mohan, P.S., Mercken, M., Farmery, M.R., Tjernberg, L.O., et al. (2005). Macroautophagy--a novel Beta-amyloid peptide-generating pathway activated in Alzheimer's disease. J. Cell Biol. 171, 87-98

Zhang, X., Li, L., Chen, S., Yang, D., Wang, Y., Zhang, X., Wang, Z., and Le, W. (2011). Rapamycin treatment augments motor neuron degeneration in SOD1(G93A) mouse model of amyotrophic lateral sclerosis. Autophagy $7,412-425$.

Zhang ,X., Chen, S., Song, L., Tang, Y., Shen, Y., Jia, L., and Le, W. (2014). MTOR-independent, autophagic enhancer trehalose prolongs motor neuron survival and ameliorates the autophagic flux defect in a mouse model of amyotrophic lateral sclerosis. Autophagy 10, 588-602. 